\title{
gु
}

\section{Disentangling the Free-Fall Arch Paradox in Silo Discharge}

\author{
S. M. Rubio-Largo, ${ }^{1}$ A. Janda, ${ }^{2, *}$ D. Maza, ${ }^{1}$ I. Zuriguel, ${ }^{1}$ and R. C. Hidalgo ${ }^{1, \dagger}$ \\ ${ }^{1}$ Departamento de Física y Matemática Aplicada, Facultad de Ciencias, Universidad de Navarra, Navarra, Spain \\ ${ }^{2}$ School of Engineering, University of Edinburgh, Edinburgh, United Kingdom
}

(Received 23 December 2014; published 11 June 2015)

\begin{abstract}
Several theoretical predictions of the mass flow rate of granular media discharged from a silo are based on the spontaneous development of a free-fall arch region, the existence of which is still controversial. In this Letter, we study experimentally and numerically the particle flow through an orifice placed at the bottom of $2 \mathrm{D}$ and $3 \mathrm{D}$ silos. The implementation of a coarse-grained technique allows a thorough description of all the kinetic and micromechanical properties of the particle flow in the outlet proximities. Though the free-fall arch does not exist as traditionally understood—a region above which particles have negligible velocity and below which particles fall solely under gravity action-we discover that the kinetic pressure displays a well-defined transition in a position that scales with the outlet size. This universal scaling explains why the free-fall arch picture has served as an approximation to describe the flow rate in the discharge of silos.
\end{abstract}

DOI: 10.1103/PhysRevLett.114.238002

PACS numbers: $45.70 . \mathrm{Mg}$

The hourglass is a paradigmatic example of the complex behavior emerging in granular flows. In these devices, the mass flow rate is mainly controlled by the orifice size, and details like particle shape and dissipation have no great significance $[1,2]$. In fact, the velocity of the grains when passing through the outlet scales only with $\sqrt{g D}$, where $g$ is the gravity acceleration and $D$ the outlet aperture [3]. This correlation has been historically associated with the existence of a singular region above the outlet, which was called the dynamic vault by Hagen in 1852 [4]. Brown and Richards revisited Hagen's idea and introduced the concept of free-fall arch as a well-defined spherical surface where the energy dissipation decreases to a minimum [5]: above this region, grains are mainly subjected to the contacting stress; below, the material is loose and moves only under the gravity action-i.e., the stress in the flow direction vanishes just at this hypothetical surface [6]. The same concept was used by other authors $[7,8]$ to develop an alternative approach, commonly known as the hourglass theory, which qualitatively predicts the mass flow rate in the discharge of a silo (see [6] for a review).

Despite the popularity that the free-fall arch idea has achieved in relation to the Beverloo correlation [1], it implies the strong formal inconsistency of assuming a spatial stress discontinuity, which is difficult to justify [9]. In addition, recent experimental efforts aimed at proving the existence of such a transition have provided inconclusive outcomes [10,11]. For instance, Vivanco et al. conducted experiments on the flow of photoelastic disks in a two-dimensional silo [10]. Examining the intensity of the force chains that appear during the discharge, they detected a well-defined boundary below which force chains were very weak. Nevertheless, this region was only identified for small orifices where the flow is intermittent and clogging is frequent [12-16]. Van Zuilichem et al. measured the density patterns in a cylindrical silo using gammaadsorption techniques [11]; they found a dilatation of the bulk close to the orifice, but a clear transition was not appreciated.

In this Letter, we present an extensive experimental and numerical study of the micromechanical properties displayed by granular media flowing through an orifice placed at the bottom of 2D and 3D silos. Using a mathematically consistent coarse-grain averaging technique (introduced by Goldhirsch [17]) we describe the velocity, density, and stress fields of the bulk close to the aperture. Hence, distinguishing between the kinetic and contact components of the stress tensor, we accurately identify a singular region where the granular flow changes its nature and scales with the size of the orifice. Furthermore, the mean acceleration shows the same scaling, which is totally compatible with the velocity profiles obtained at the silo outlet $[3,18]$. As a result, the mass flow rate correlations previously introduced [1-3] can be consistently derived.

The experimental setup consists of a quasi-twodimensional silo built with two glass sheets (height $800 \mathrm{~mm}$ and width $200 \mathrm{~mm}$ ) separated by a steel frame which also conforms the lateral walls. The gap between the glass sheets is slightly larger than the particles diameter $\left(d=2 r_{p}=1.0 \mathrm{~mm}\right)$ so the beads can only arrange themselves in a single layer. The particles flow out through a horizontal slit of a tunable aperture $D=2 R$ located at the flat bottom of the silo. The discharge process is recorded by means of a high-speed camera. The videos were analyzed using image processing techniques allowing for a precise determination of the instantaneous position $\vec{r}$ and velocity $\vec{v}$ of each particle. A more detailed description of the 
experimental setup and image analysis techniques can be found in [15] and [3], respectively.

In order to get access to the micromechanical properties of the system and extend the experimental findings in two dimensions to a more realistic 3D silo, we have resorted to numerical simulations. A hybrid CPU-GPU discrete element algorithm $[19,20]$ has been developed for a system of spheres with diameter $d=2 r_{p}=\frac{1}{32} \mathrm{~m}$ and density $\rho=7520 \mathrm{~kg} / \mathrm{m}^{3}$. The contact interaction has been simulated using a linear-dashpot model with normal and tangential elastic constants, $k_{n}=10^{8} \mathrm{~N} / \mathrm{m}$ and $k_{t}=\frac{2}{7} k_{n}$, respectively. The ratio between normal and tangential damping coefficients was taken as $\nu_{n} / \nu_{t}=3$ with $\nu_{n}=10^{3} \mathrm{~s}^{-1}$. Accordingly, the equations of motion were integrated with $\Delta t=10^{-6} \mathrm{~s}$ (more details can be found in $[18,20])$. A flat-bottom cylindrical container is implemented with a radius $W=32 r_{p}$ and walls with equivalent mechanical properties to those of the particles. We generate a granular column from a granular gas of $N=262144$ spheres that are initially located within the cylinder at random locations. Then, they settle under the effect of gravity and are allowed to leave the system through a circular outlet centered at the bottom of the column. After certain time, a steady state is reached with a column height of around $h_{\max }=112 d$.

The kinetic and dynamic properties of the particle flow have been examined using a consistent coarse-graining methodology [17,21]. First, we have accessed to the exact positions and velocities of the particles in the experiments and numerical simulations. Then, the coarse-grained mass density of a granular flow, $\rho(\vec{r})$, at time $t$ is obtained as $\rho(\vec{r})=\sum_{i=1}^{N} m_{i} \phi\left(\vec{r}-\vec{r}_{i}(t)\right)$ where the sum runs over all particles within the system and $\phi\left(\vec{r}-\vec{r}_{i}(t)\right)$ is an integrable coarse-graining function [17]. In the same way, the coarsegrained momentum density function $P(\vec{r}, t)$ can be defined by $P(\vec{r}, t)=\sum_{i=1}^{N} m_{i} \vec{v}_{i} \phi\left(\vec{r}-\vec{r}_{i}(t)\right)$, where $\vec{v}_{i}$ represents the velocity of particle $i$. The macroscopic velocity field $\vec{V}(\vec{r}, t)$ is then defined as the ratio of momentum and density fields, $V(\vec{r}, t)=P(\vec{r}, t) / \rho(\vec{r}, t)$.

The macroscopic stress field $\sigma_{\alpha \beta}$ can be decomposed into a contact stress field $\left(\sigma_{\alpha \beta}^{c}\right)$ related with particleparticle interactions, and a kinetic stress field $\left(\sigma_{\alpha \beta}^{k}\right)$ which depends on the velocity fluctuations of a single particle with respect to the macroscopic flow [17]. The contact stress reads

$$
\sigma_{\alpha \beta}^{c}=-\frac{1}{2} \sum_{i=1}^{N} \sum_{j=1}^{N c_{i}} f_{i j \alpha} r_{i j \beta} \int_{0}^{1} \phi\left(\vec{r}-\vec{r}_{i}+s \vec{r}_{i j}\right) d s
$$

where the first sum runs over all the particles and the second accounts for the $N c_{i}$ contacting particles of $i$. Moreover, $\vec{f}_{i j}$ accounts for the force exerted by particle $j$ on particle $i$ and $\vec{r}_{i j} \equiv \vec{r}_{i}-\vec{r}_{j}$. The kinetic stress reads

$$
\sigma_{\alpha \beta}^{k}=-\sum_{i}^{N} m_{i} \vec{v}_{i \alpha}^{\prime} \vec{v}_{i \beta}^{\prime} \phi\left(\vec{r}-\vec{r}_{i}\right)
$$

where $\vec{v}_{i}^{\prime}(\vec{r}, t)=\vec{v}_{i}(t)-\vec{V}(\vec{r}, t)$.

Based on these definitions, we have implemented a postprocessing tool to resolve the kinetic fields in the 2D (experimental) and 3D (numerical) discharge processes. In complement, all the micromechanical properties of the simulated flow have been explored in detail. We use a Gaussian coarse-graining function, $\phi(\overrightarrow{\mathcal{R}})=$ $\left[1 /(\sqrt{2 \pi} w)^{3}\right] \exp \left(-|\overrightarrow{\mathcal{R}}|^{2} / 2 w^{2}\right)$, with $\overrightarrow{\mathcal{R}}=\vec{r}-\vec{r}_{i}$, and where the coarse-graining scale has always been kept as $w=r_{p}$. The fields are explored at a region close to the outlet for a range of apertures $8 r_{p}<R<26 r_{p}$, thus guaranteeing the absence of clogging. In all cases, the temporal averaging of the variables is computed along the whole measurement time. In the 3D silo, cylindrical averaging around the vertical axis is also performed. Thus, the magnitudes are displayed as 2D azimuthal averages where $r$ represents the radial distance to the center of the outlet.

First, we show the spatial dependence of the vertical velocity, $v_{z}$, and volume fraction fields within the 2D and 3D silos (Fig. 1). In both situations the coarse-graining magnitudes display monotonic behavior with height: When approaching the orifice, velocity increases and volume fraction decreases. For completeness we have checked that the kinetic profiles at $z=0$ are coherent with previous results $[3,18]$.

Next, we investigate the micromechanical details of the particle flow by focusing on the changes in the mean kinetic and contact tensors. The spatial profile of the contact pressure, defined as the trace of $\sigma_{\alpha \beta}^{c}$, is illustrated in Fig. 2(a). As the height is reduced, the contact stress monotonically decreases due to dilatation of the bulk [11],
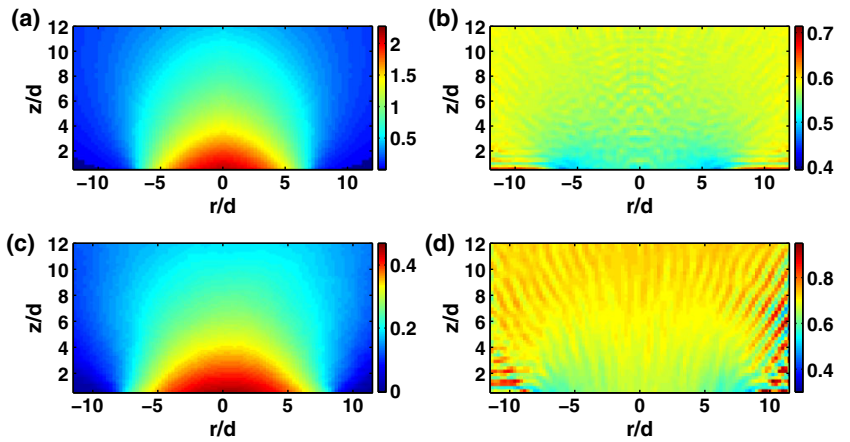

FIG. 1 (color online). Vertical velocity fields in (a) 3D numerical and (c) 2D experimental silos. Solid fraction fields in (b) numerical and (d) experimental silos. In all cases $R=16 r_{p}$ and a Gaussian coarse-graining function $\phi(\overrightarrow{\mathcal{R}})$ with $w=r_{p}$ has been used. In the figures, the vertical coordinate, $z$, indicates the height from the bottom of the silo, and the horizontal coordinate, $r$, the distance to the center of the silo. 

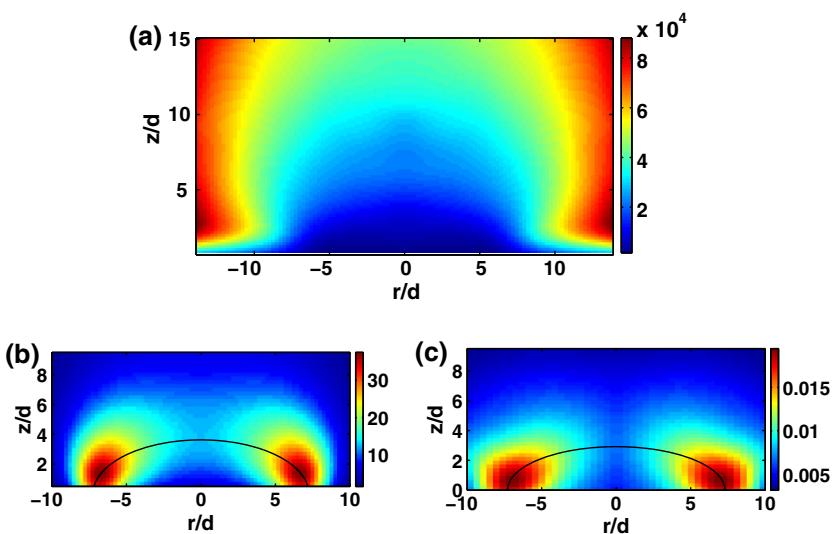

FIG. 2 (color online). (a) Contact pressure $\operatorname{tr}\left[\sigma_{\alpha \beta}^{c}\right]$ profile obtained from simulations. At the bottom, spatial profiles of the (b) numerical and (c) experimental kinetic pressure. The line is a parabolic fitting of the local maximum position. All graphs correspond to $R=16 r_{p}$, and the data have been calculated with a Gaussian coarse-graining function $\phi(\overrightarrow{\mathcal{R}})$ and $w=r_{p}$.

which is compatible with the density fields shown in Fig. 1. Additionally, the correlation between the traction transmission and the velocity fluctuations can be outlined examining the kinetic stress. Importantly, this magnitude is also accessible for the experimental case as it only depends on the fluctuations of the particle's velocity respect to the macroscopic flow. As in the case of $\sigma_{\alpha \beta}^{c}$, we quantify the strength of the kinetic pressure with the trace of $\sigma_{\alpha \beta}^{k}$ [Eq. (2)]. The obtained values [Figs. 2(b)-2(c)] are several orders of magnitude smaller than the contact pressure, but the spatial pattern looks very different. Both experimental and numerical outcomes show nonmonotonic trends indicating the existence of a region where the kinetic pressure is maximum. Note that the existence of a low-pressure cavity above the outlet was already observed by implementing a plastic rheology in a 2D Navier-Stokes solver following the $\mu(I)$ rheology [22].

For sake of simplicity, we focus on the evolution of the contact and kinetic pressure along the vertical direction $z$ at the center of the orifice. As evidenced in Fig. 2(a), the contact pressure decays monotonically when approaching the bottom of the silo [Fig. 3(a)]. Remarkably, the gradient of contact pressure is constant [typically of the same order of magnitude than the gravity body force $\rho g \phi(r, z)]$ and nearly independent on the outlet size. Consequently, a region resembling a free-fall arch (that should scale with $R$ ) cannot be inferred from these findings. Meanwhile, the kinetic pressure shows a nonmonotonic behavior [Fig. 3(b)]. Far from the outlet, particles follow a global macroscopic laminar flow where advection dominates their movements and, as consequence, the kinetic pressure is negligible. As the height decreases, the kinetic pressure grows exponentially until it reaches a well-defined maximum the location of which, $z_{c}$, depends on $R$. Indeed, the kinetic pressure profiles can be collapsed normalizing the
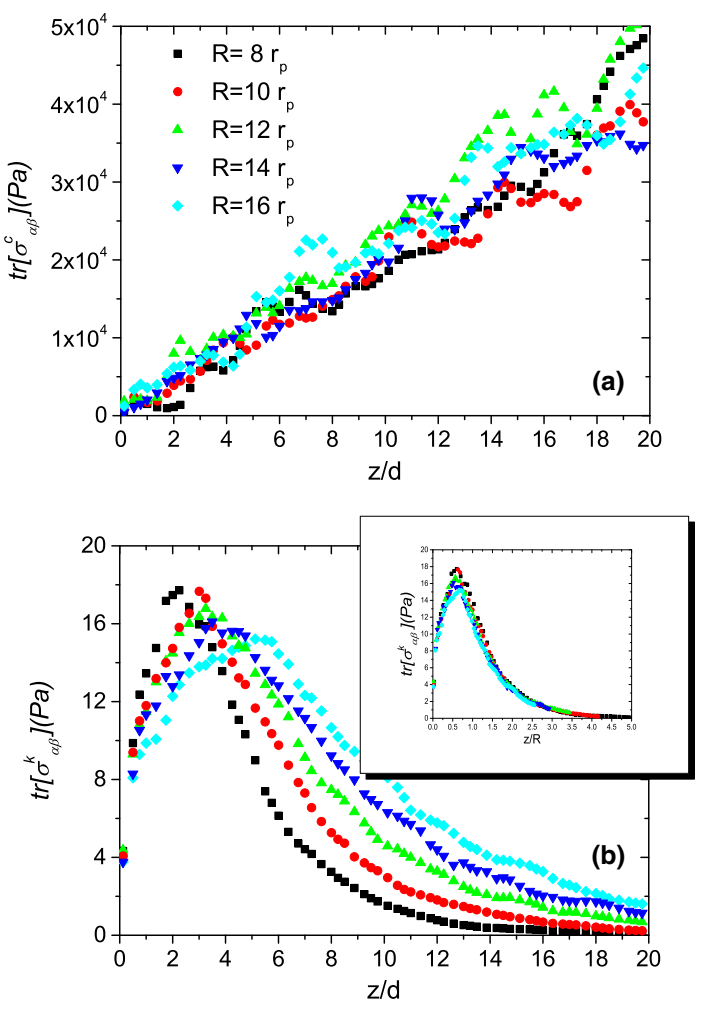

FIG. 3 (color online). Vertical profiles of (a) contact pressure, $\operatorname{tr}\left[\sigma_{\alpha \beta}^{c}\right]$, and (b) kinetic pressure, $\operatorname{tr}\left[\sigma_{\alpha \beta}^{k}\right]$, at the center of the silo ( $r=0$ ), for different outlet sizes $R$ as indicated in the legend. The inset shows the data collapse when normalizing the vertical coordinate $z$ with the size of the outlet $R$.

vertical coordinate with $R$ [Fig. 3(b), inset]. Hence, although the kinetic stress is much smaller than the contact stress, the scaling of its spatial profile with $R$ suggests its importance in the discharge process.

In order to unveil the relationship between the spatial dependence of kinetic pressure and the shape of velocity profiles at the outlet, Fig. 4 illustrates the vertical position, $z_{c}$, for which the kinetic pressure is maximum, as a function
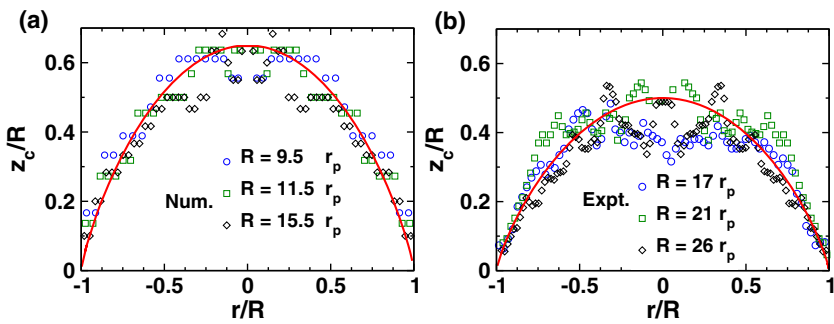

FIG. 4 (color online). Position of the local maximum of the kinetic stress as a function of the radial distance for (a) 3D (numerical) and (b) 2D (experimental) silos. All the data have been collapsed normalizing the vertical and horizontal distances with the radius of the outlet. The red lines correspond to a parabolic fit which can be associated to the modified concept of the free-fall arch. 
of the radial distance, $r$. All curves obtained for different outlet sizes can be collapsed by rescaling both $z_{c}$ and $r$ by the outlet size, $R$. More importantly, the collapsed data can be fitted by a parabolic dome. Although the height of these domes seems to depend on the dimensionality, the collapse prompts us to reframe the concept of free-fall arch as the region where the collisional part of the stress tensor starts to diminish and the dynamics becomes gradually dominated by the external field. This picture contrasts with the traditional view of a location where the velocity is negligible and grains start a free fall.

The unsuitability of this historical idea can be confirmed by examining the effective acceleration profiles $a_{\text {eff }}=$ $d v_{z} / d t$. The experimental and numerical outcomes of $a_{\text {eff }}(z)$ at the center of the orifice $(r=0)$ and along the vertical direction $(h=z / R)$ are shown in Fig. 5. The values of $a_{\mathrm{eff}}(z)$ were calculated finding the finite difference of the velocity vector of a single particle in two consecutive times (in simulations $\Delta t_{s}=t_{d} / 8$, and in experiments $\Delta t_{e} \approx t_{d} / 4$, where $t_{d}=\sqrt{2 d / g}$. The data shown correspond to averages over square (2D) or cubic (3D) regions of width $\Delta=2 r_{p}$. Similar to the kinetic stress, the vertical acceleration profiles collapse into a single master curve when $z$ is normalized by $R$. Remarkably, for both the 2D and 3D silos, the collapse is very robust and the curve trends are similar: As particles move down, acceleration increases, and it only equals the gravity at the outlet. For comparison, the acceleration profiles expected from the traditional

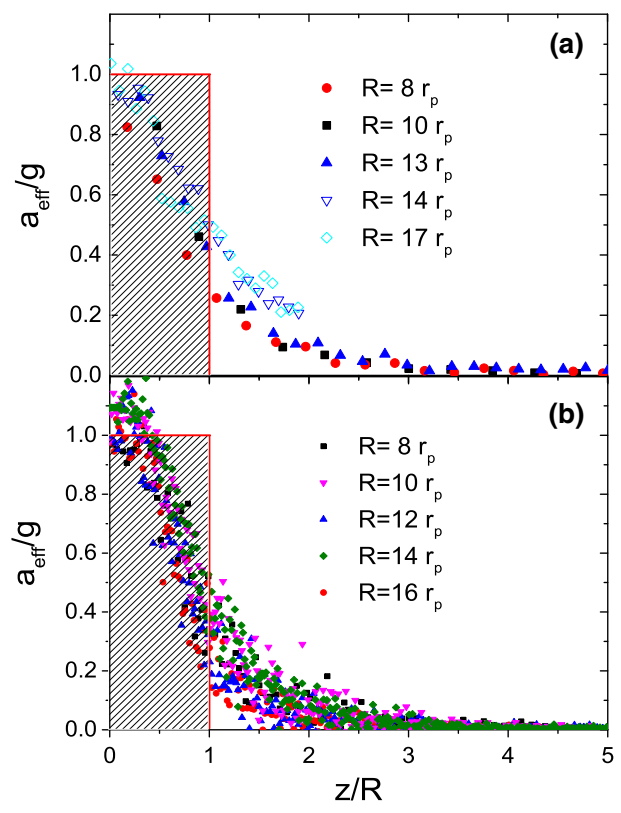

FIG. 5 (color online). Effective acceleration normalized by the gravity as a function of the normalized vertical distance $z / R$ in (a) a $2 \mathrm{D}$ experimental silo and (b) a $3 \mathrm{D}$ numerically simulated silo. In both cases, the measurements have been performed at $r=0$. The shadow region corresponds to the Heaviside function compatible with the historical idea of the free-fall arch. picture of the free-fall arch are illustrated by a Heaviside function (shadowed region in Fig. 5). Incidentally, acceleration values slightly greater than $g$ seem to appear close to the aperture. We speculate that the origin of such an excess of acceleration is caused by particles being ejected downwards, which indicates that the system compression is still significant at this location.

The observations reported in Fig. 5 seem to challenge the validity of the scaling $v_{c}=\sqrt{2 \gamma g R}$, where $\gamma=1.07 \pm$ 0.015 was a fitting parameter experimentally obtained from the dependency of $v_{c}$ on $R$ [3]. Far from that, the selfsimilarity of the acceleration profiles is the key that provides the argument to analytically calculate the scaling function $v_{c}$ as follows. First, the vertical evolution of the acceleration is generally written as $\mathcal{F}(h)=a(h) / g$, where $\mathcal{F}(h)$ is a self-similar function of $h=z / R$. After a simple change of variables, this expression can be rewritten as $v d v=g R \mathcal{F}(h) d h$. Then, we integrate assuming that the silo expands over the upper half plane, with the outlet centered at the origin,

$$
\int_{0}^{v_{c}} v d v=\frac{v_{c}^{2}}{2}=g R \int_{\infty}^{0} \mathcal{F}(h) d h=\gamma g R .
$$

Straightforwardly $v_{c}=\sqrt{2 \gamma g R}$, where $\gamma$ accounts for the area under the curves of Fig. 5. We have obtained $\gamma=1.10$ and $\gamma=1.09$ for the 2D experimental and 3D numerical cases, respectively. Following the original hypothesis of the free-fall arch, the value of $\gamma$ would be equal to 1 . As a consequence, the scaling ansatz introduced in [3] is totally justified, as it is founded on the self-similarity of the dynamical fields inside the silo. Indeed, it can be stated that the scaling $v_{c} \propto \sqrt{g R}$ is imposed by the fact that $R$ is the only relevant length scale in the system, and the asymptotic evolution of the acceleration towards the gravity value $g$.

In summary, our findings disentangle the paradoxical ideas associated with the free-fall arch in the discharge of a silo, which has historically served to justify the dependence of the flow rate on the outlet size. As suggested earlier [4], there exists a singular region above the outlet where certain dynamical features decide the resulting velocity profile. Nevertheless, by no means does this region meet the naive picture of an arch where particles lose all their kinetic energy and start to fall freely under gravity. In the same way, discontinuities in the stress field $[5,7,8]$ have not been identified either. We have shown that the stress decomposition into contact and kinetic parts [17] provides a suitable theoretical framework to distinguish between the different role of force chains and velocity fluctuations. The contact pressure displays a constant decrease as one approaches the exit. This behavior is practically independent of the aperture size, indicating that the pressure inside the silo does not have a one-to-one relationship with the velocity profile at the exit, as was already suggested in [23]. 
Meanwhile, the kinetic pressure displays a well-defined maximum near the outlet that defines a transition surface. The scaling of this surface with the size of the aperture links the microscopic dynamics observed for the discharge process with the stress fields, suggesting that the only relevant length scale in the problem is $R$. At this point, the independence of contact pressure with $R$ might be a surprising result. However, it is important to remark that all the fields examined represent a continuum description of our granular system in stationary conditions. In fact, the advective motion of a representative volume element (RVE) is governed by the momentum balance in terms of the gravity, the gradient of contact pressure, and the dissipative shear stress. For symmetry reasons, the shear stress diminishes at the center of the silo and maximum values of the vertical velocity $V_{\max }$ are developed. As the gradient of contact stress is constant, regardless the outlet size, it is plausible to accept that $V_{\max }$ is controlled by the distance from the RVE to the border of the orifice, which is in agreement with the role of $R$ as the only characteristic length of the discharge process. Finally, based on the acceleration profiles collapsed with $R$, we explain the scaling of the exit velocity with $\sqrt{g R}[3,18]$ as well as the traditional mass flow rate correlations used in engineering $[1,2]$.

This work has been supported by Projects No. FIS201126675 and No. FIS2014-57325 Ministerio de Economía y Competitividad (Spanish Government) and PIUNA (Universidad de Navarra). S. M. R. thanks Asociación de Amigos de la Universidad de Navarra for a scholarship. D. M. thanks MP1305 Flowing Matter COST Action.

\footnotetext{
*A.Janda@ed.ac.uk †raulcruz@unav.es
}

[1] W. A. Beverloo, H. A. Leniger, and J. J. Van de Velde, Chem. Eng. Sci. 15, 260 (1961).

[2] C. Mankoc, A. Janda, R. Arévalo, M. Pastor, I. Zuriguel, A. Garcimartin, and D. Maza, Granular Matter 9, 407 (2007).
[3] A. Janda, I. Zuriguel, and D. Maza, Phys. Rev. Lett. 108, 248001 (2012).

[4] B.P. Tighe and M. Sperl, Granular Matter 9, 141 (2007).

[5] R. C. Brown and J. C. Richards, Nature (London) 191, 458 (1961).

[6] R. M. Nedderman, Statistics and Kinematics of Granular Materials (Cambridge University Press, Cambridge, England, 1992).

[7] S. B. Savage, Int. J. Mech. Sci. 9, 651 (1967).

[8] J. F. Davidson and R. M. Nedderman, Trans. Inst. Chem. Eng. 51, 29 (1973).

[9] D. L. Henann and K. Kamrin, Phys. Rev. Lett. 113, 178001 (2014).

[10] F. Vivanco, S. Rica, and F. Melo, Granular Matter 14, 563 (2012).

[11] D. J. Van Zuilichem, N. D. Van Egmond, and J. G. De Swart, Powder Technol. 10, 161 (1974).

[12] K. To, P. Y. Lai, and H. K. Pak, Phys. Rev. Lett. 86, 71 (2001).

[13] I. Zuriguel, D. R. Parisi, R. C. Hidalgo, C. Lozano, A. Janda, P. A. Gago, J. P. Peralta, L. M. Ferrer, L. A. Pugnaloni, E. Clément, D. Maza, I. Pagonabarraga, and A. Garcimartín, Sci. Rep. 4, 7324 (2014).

[14] I. Zuriguel, A. Janda, A. Garcimartín, C. Lozano, R. Arévalo, and D. Maza, Phys. Rev. Lett. 107, 278001 (2011).

[15] A. Janda, I. Zuriguel, I. A. Garcimartín, L. A. Pugnaloni, and D. Maza, Europhys. Lett. 84, 44002 (2008).

[16] C. C. Thomas and D. J. Durian, Phys. Rev. Lett. 114, 178001 (2015).

[17] I. Goldhirsch, Granular Matter 12, 239 (2010).

[18] D. Maza, A. Janda, S. M. Rubio-Largo, I. Zuriguel, and R. C. Hidalgo, AIP Conf. Proc. 1542, 674 (2013).

[19] J. Owens, M. Houston, D. Luebke, S. Green, J. Stone, and J. Phillips, Proc. IEEE 96, 879 (2008).

[20] R. C. Hidalgo, T. Kanzaqui, T. Alonso-Marroquin, and S. Luding, AIP Conf. Proc. 1542, 169 (2013).

[21] T. Weinhart, A. R. Thornton, S. Luding, and O. Bokhove, Granular Matter 14, 531 (2012).

[22] L. Staron, P.-Y. Lagre, and S. Popinet, Phys. Fluids 24, 103301 (2012).

[23] M. A. Aguirre, J. G. Grande, A. Calvo, L. A. Pugnaloni, and J.-C. Géminard, Phys. Rev. Lett. 104, 238002 (2010). 